\title{
Desarrollo de nuevos procesos de operación y administración, soportados en innovación organizacional y herramientas de tecnologías de información para una empresa del sector automotor
}

\section{Development of new operation and management processes, supported by organizational innovation and information technology tools for a company in the automotive sector}

\author{
M. A. Vélez, C. M. Sánchez, H. S. Fuquen
}

Recibido: marzo 12 de 2019 - Aceptado: junio 15 de 2020

\begin{abstract}
Resumen-El presente artículo pretende mostrar cómo el crecimiento del mercado de las motocicletas ha generado la necesidad de innovar la arquitectura empresarial de las ensambladoras y las redes de distribución y comercialización para obtener economías de escala enfocadas a mejorar la productividad y competitiva de las compañías. En el texto se realizará una breve descripción de los conceptos relacionados con la metodología para transformación digital de los negocios, así como una descripción de la aplicación en el proyecto de desarrollo tecnológico y organizacional implementado en FANALCA S.A y la red de concesionarios relacionados con la marca de motocicletas HONDA.
\end{abstract}

Palabras clave - Arquitectura empresarial, transformación digital de negocios, motocicletas

${ }^{1}$ Producto derivado del proyecto calificado de innovación "Desarrollo de nuevos procesos de operación y gerencia, soportados en el desarrollo organizacional y herramientas de tecnologías de información para FANALCA S.A.”. Presentado a la convocatoria de Beneficios Tributarios de COLCIENCIAS.

M. A. Vélez, Coinvestigador del Proyecto en FANALCA S.A, Bogotá, Colombia, email: mvelez@fanalca.com.

C. M. Sánchez, Investigador Asociado Reconocido por COLCIENCIAS, grupo de investigación COLINNOVACION, Bogotá, Colombia, email: csanchez@colinnovacion.com.

H. S. Fuquen, Investigador junior Reconocido por COLCIENCIAS, grupo de investigación COLINNOVACION, Bogotá, Colombia, email: hfuquen@colinnovacion.com.

Como citar este artículo: Vélez M. A, Sánchez C. M., Fuquen, H. S., Desarrollo de nuevos procesos de operación y administración, soportados en innovación organizacional y herramientas de tecnologías de información para una empresa del sector automotor, Entre Ciencia e Ingeniería, vol. 14, no. 27 , pp. 29-34, enero-junio 2020. DOI: https://doi.org/10.31908/19098367.1741.

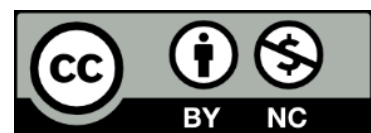

Attribution-NonCommercial 4.0 Intenational (CC By-NC 4.0)
Abstract - The following article shows how the growing motorcycle market has created the need to innovate the business architecture of the assemblers and the distribution and commercialization networks, in order to obtain economies of scale and to improve productivity and competitiveness. The text provides a brief description of concepts relating to the digital business transformation methodology, as well as a description of its application in the technological and organizational development project in FANALCA S.A and the HONDA Motorcycles dealership network.

Keywords- Enterprise architecture, digital business transformation, motorcycles.

\section{INTRODUCCIÓN}

${ }^{1}$ uso de las motocicletas ha venido incrementándose de - manera importante a lo largo de las últimas décadas, haciendo que variables como la producción y la comercialización de estos vehículos presentaran crecimientos sin precedentes dentro del sector industrial en Colombia. "Mientras en 1998 rodaban menos de un millón de motocicletas, en 2016 superaron los 7,2 millones" [1]. Este escenario ha presentado un reto al interior de las empresas ensambladoras, de tal forma que su crecimiento evidencie economía de escala. Es allí donde la innovación presenta un factor esencial para rediseñar la forma en que opera una empresa. La innovación definida en el Manual de Oslo como: "Un Nuevo o mejorado producto o proceso (o una combinación de estos) que difiere significativamente de previos productos o procesos y que se encuentran disponibles para usuarios potenciales (en el caso de los productos) o en uso por parte de la empresa (en caso de procesos)" [2]. La innovación en procesos, en la edición 3 del manual incluía los Sistemas de Información y comunicaciones. Así mismo, dentro de las tipologías de innovación la edición 3, se reconocía la innovación organizacional, que en las definiciones de la Edición 4, se cataloga en actividades de gestión y administración, particularmente dentro de las actividades de gestión estratégica [2]. Estas incluyen la 
conceptualización integral de la organización a través de la arquitectura empresarial basada en una visión futura con el objetivo de favorecer la mejora continua, el análisis de impacto por la modificación de algunos de sus componentes, las decisiones estratégicas o cualquier acción que requiera la visión de la organización como un todo. La innovación propuesta que se describe a continuación fue objeto de reconocimiento por parte de COLCIENCIAS como proyecto de innovación empresarial.

El presente artículo surge del programa de innovación organizacional, cuyo objetivo fue construir un nuevo esquema de arquitectura empresarial, soportado en el diseño e incorporación de arquitecturas basadas en procesos, tecnologías de información y estructuras organizacionales, que le permitieran a FANALCA y su grupo de distribuidores incrementar la eficacia de su cadena de valor.

FANALCA S.A. es una empresa del sector metalmecánico que hace más de 60 años inició el negocio automotriz, suministrando partes metálicas estampadas para las principales ensambladoras de vehículos y camiones del Grupo Andino. En 1981 FANALCA S.A. se convirtió en fabricante y distribuidora exclusiva de Honda en Colombia., "con una red de más de 350 puntos de venta ubicados en toda la geografía nacional" [1] en los cuales, además de la distribución y el servicio técnico, se tiene un proceso de sensibilización de conductores de motocicleta, a través de Simuladores de Conducción Honda, ubicados en la red de concesionarios [1].

\section{ESTADO DE LA TÉCNICA}

\section{A. Transformación digital de los negocios (DBT)}

La transformación digital de los negocios es definida por Gartner como "el proceso de explotación de las tecnologías digitales y las capacidades de soporte para crear un nuevo modelo de negocio digital sólido" [3]. Innovation \& Entrepreneurship Business School va más allá al recalcar que el cambio que incluye este concepto no es sólo tecnológico "sino que lleva consigo nuevas aptitudes tanto en las personas como en la reinvención de organizaciones que afectan al mercado global tradicional. No está enfocada a la tecnología utilizada, sino en utilizarla para lograr los objetivos planteados. La transformación digital va ligada con los objetivos y estrategias empresariales" [4].

La DBT (por sus siglas en inglés: Digital Business Transformation) a lo largo de la organización, garantiza la presencia de beneficios como aumento de los ingresos, eficiencia en los procesos y mejora de la cultura de innovación organizacional [5]. Por tanto, se define el DBT como un método integral para la estructuración de proyectos de transformación, mejora de los procesos, reducción de ineficiencias, escalabilidad de los sistemas, permitiendo tener mejores herramientas de análisis.

La arquitectura tradicional de transferencia de datos vía web ha evolucionado hacia el concepto "many to many", donde la capacidad de transmisión y recepción es limitada exclusivamente por la envergadura física de los sistemas hardware de comunicación de las empresas. Expandiendo las capacidades de la transferencia de información por Internet, permitiendo la transmisión eficiente y sincronizada de datos a través de los diferentes eslabones pertenecientes a una cadena de valor en una arquitectura empresarial. Ampliando el concepto de DBT, no solo la redistribución de procesos y sinergia tecnológica, sino a la posibilidad de estructurar procesos de negocios que facilite la interacción de los componentes de una cadena de valor [6].

Las compañías están valorando fuertemente incorporar la DBT, como puede verse en la Fig. 1 el 89\% de las compañías encuestadas adoptaron o tienen planes de adoptar estrategias digitales por primera vez y en el caso específico de las empresas de manufacturas el $87 \%$ lo han hecho o tienen planes específicos de hacerlo [7]. Esto lleva a la conclusión de que la DBT será un factor competitivo en los próximos años.

DBT incluye diferentes ámbitos a desarrollar dentro de una estrategia digital, una gestión de la transformación, así como tecnologías digitales y empresariales integradas [8].

\section{1) Estrategia Digital}

Son métodos de descubrimiento interdisciplinario para diseñar y priorizar las oportunidades de transformación. Organizándolos en un mapa de ruta (roadmap) para la evolución digital. Alineando las iniciativas digitales con la estrategia de negocios de la organización.
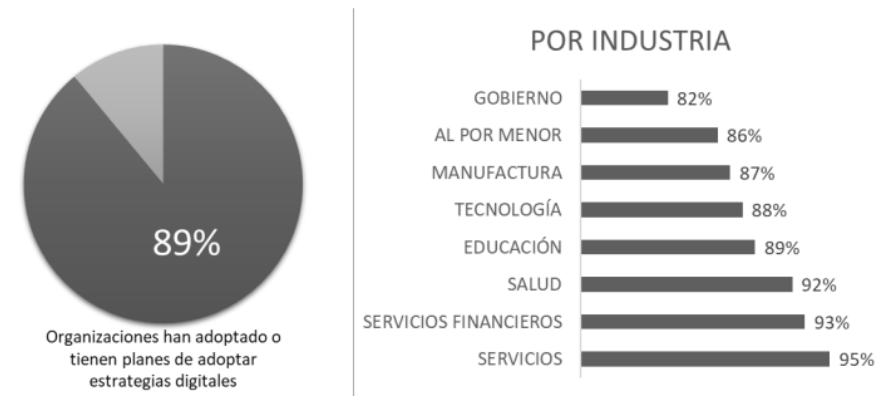

Fig. 1. Prioridad de los Negocios Digitales [7].

\section{2) Gestión de la Transformación}

Combina la gestión de procesos, de las personas y del cambio en la organización. Incluye además la gestión del valor, mediante modelos cuantitativos con el objetivo de capturar los beneficios económicos y operativos de las iniciativas.

\section{3) Tecnologías Digitales y Empresariales Integradas}

Combina la gestión de procesos, de las personas y de aplicaciones específicas, combinando nuevas plataformas de intercambio electrónico, movilidad, redes sociales y Big data; con una adaptación e integración de los sistemas empresariales para soportar los nuevos procesos.

\section{B. Arquitectura Empresarial (AE)}

La Arquitectura Empresarial se define de acuerdo con el Centro de Investigación de Sistemas de Información del MIT como "la lógica organizativa para los procesos de negocio y las capacidades de tecnología informática, que reflejan los requisitos de integración y estandarización del modelo operativo de la empresa" [9]. Esta conceptualización integral de la organización permite proyectar la empresa a una arquitectura empresarial futura, como una visión de la organización en cada uno de sus componentes entre los cuales se encuentran: personas, procesos, tecnología y valor. 


\section{Modelo Único Replicable (MUR)}

Es una metodología para el modelado y evolución de modelos corporativos. El modelo incluye sistemas de información, procesos, tecnología de operación e integración y analíticos.

Cuando se refiere a modelos corporativos se enmarca en un grupo empresarial o múltiples unidades de negocio a lo largo de una cadena de valor, encontrando sinergias, es decir buscando la estandarización de procesos existentes y nuevos, así como compartir las mejores prácticas entre la mayor cantidad de empresas o unidades de negocio posibles. De esta forma, se pueden detectar e implementar procesos de negocios que podrían centralizarse permitiendo reducción de costos y eficiencias en las diferentes áreas de la organización.

\section{Centro de Transformación de Tecnología (CTT)}

Es un grupo interdisciplinario que se crea al interior de las organizaciones para garantizar que la apropiación de la metodología de transformación digital de los negocios, supervisión, implementación y evolución del MUR y desarrollo de los servicios al interior de la organización en disciplinas de gestión de valor, gestión de proyectos, gestión de personas, gestión de procesos y gestión de tecnología.

\section{E. Minería de Datos}

La minería de datos puede ser definida como un proceso sistemático de exploración útil, para encontrar información potencial y características comunes dentro de un conjunto de datos de gran extensión [10]. Existen ejemplos de aplicaciones de negocio que incluyen análisis del comportamiento del consumidor, mercadeo, gestión de clientes e inteligencia de negocios, es la forma en que la minería de datos provee una metodología para recolectar, analizar e identificar información útil [11].

La minería de datos es indispensable en la estandarización de proyectos, debido que provee una estructura que permite almacenar las características de los proyectos para que pueda ser replicado más adelante a una organización por parte de cualquier persona [12].

En el proceso de migración de datos, es necesaria la consolidación de la información en una base de datos estructurada y parametrizable para la extracción de patrones, con el objetivo que posteriormente se unifique la información dispersa a migrar. El nombre de este tipo de bases de datos propia de la migración de información es Data Warehouse, definidas como repositorios de información recolectada por múltiples fuentes, almacenada bajo un esquema unificado y usualmente ubicado en un solo lugar. Los Data Warehouse se construyen por procesos definidos tales como limpieza de datos, integración de datos, transformación de datos, carga de datos y periódicamente actualización de los datos. [13].

Las bases de datos actuales tienen la capacidad de almacenar grandes volúmenes de datos, con características estructurales para facilitar la minería de datos o el acceso inmediato a información específica. Los Data Warehouses se caracterizan por consolidar la información en es espacios virtuales multidimensionales definidos como cubos de datos o "Data Cubes", facilitando los procesos de minería de datos [13].

Comúnmente el uso de las bases de datos para transacciones de información es de mayor dominio por parte de los profesionales y los usuarios de esta clase de sistemas, sin embargo, según la aplicación en la gestión de la información de las bases de datos, existen diferencias entre dos clases de sistemas para negocios digitales: por un lado se encuentran las transacciones y procesamiento ágil, y en segundo lugar los sistemas de análisis y decisión, denominados respectivamente OLTP (OnLine Transaction Processing) y OLAP (OnLine Analytical Processing).

Los sistemas OLTP o Sistemas de Bases de Datos, cubren la mayor cantidad de operaciones diarias de una organización tales como compras, inventario, manufactura, banca, pago de impuestos, registros, y contabilidad. Mientras que los OLAP o Data Warehouse, apoyan a los usuarios en los procesos de análisis de datos y toma de decisiones. Dichos sistemas pueden organizar y presentar los datos procesados en diferentes formatos para suplir las diversas necesidades de los usuarios [13]

A continuación, se relacionan las características que diferencian los sistemas de almacenamiento OLAP y OLTP:

Orientación de Usuarios y Sistemas: un sistema OLTP es un sistema orientado al consumidor y es usado para transacciones y procesamiento ágil por los empleados, clientes y profesionales expertos en TI. Los OLAP están orientados al mercado y son usados para el análisis de datos por trabajadores de conocimiento, incluyendo administradores, ejecutivos, y analistas.

Contenido de Datos: un OLTP administra datos actuales que, normalmente, se presentan de forma detallada para la toma de decisiones. Mientras que los OLAP administran gran cantidad de datos históricos, proporcionan facilidades para sintetizar y depurar, y almacenan información de diferentes niveles de dispersión de la información. Estas características permiten que los datos puedan ser usados para la toma de decisiones.

Diseño de Bases de Datos: un sistema OLTP usualmente adopta modelos Entidad-Relación y diseño de BD orientadas a aplicaciones. Un OLAP normalmente adopta como modelos tipo star o snowflake y diseño de BD orientado a objetos.

Un OLTP se enfoca principalmente en los datos actuales con empresas o departamentos, sin referirse a datos históricos o datos de diferentes fuentes. Por el contrario, OLAP permite procesar múltiples esquemas de bases de datos, en paralelo con el proceso evolutivo de una organización. Así mismo OLAP permite la integración de información de diferentes repositorios de datos, porque los datos de este tipo de sistema se almacenan en varios medios de almacenamiento.

Patrones de Acceso: en el caso de OLTP consiste en transacciones pequeñas, dichos sistemas requieren control concurrente y mecanismos de recuperación. No obstante, el acceso a los sistemas OLAP son mayormente de procesos de lectura, siendo en la mayoría de los casos lecturas complejas de información estructurada.

\section{F. Industria de motocicletas y la distribución del producto.}

Para el sector automotor en general, las principales fuentes de ingresos son la venta única de vehículos, así como autopartes, mantenimiento entre otros productos y servicios del mercado posventa [14]. El proceso de comercialización de los vehículos, en el caso de la industria de motocicletas, presenta el mismo modelo que en el sector de automóviles. 
Llegar al cliente final implica el desarrollo de una red de concesionarios, no obligatoriamente pertenecientes a la marca [15], pero que en ocasiones tendrían convenios de exclusividad, para realizar distribución y venta tanto al por mayor como al por menor de motocicletas, repuestos y accesorios para el vehículo, ropa y accesorios para el usuario [16] e incluso combustible [17].

Durante las últimas décadas del siglo $\mathrm{XX}$, la venta de un vehículo nuevo permaneció relativamente inalterado: un vendedor guiaba al cliente a través de un proceso de adquisición de hasta 18 pasos [18]. Actualmente, los fabricantes además de la distribución de sus productos toman información de mercado de las fuentes de datos de los distribuidores [15], que se ha transformado en una de las fuerzas externas que configuran tanto la estructura como las estrategias de esta industria [19], razón por la cual se ha hecho fundamental mantener entre distribuidor y fabricante canales y procesos de comunicación adecuados.

Según los análisis de tendencias, el relacionamiento entre el cliente final y el fabricante de vehículos se mantendrá a través de los distribuidores; el concesionario seguirá siendo un punto de contacto importante para los prospectos de compradores [20]. Adicionalmente, en las tendencias de futuro muestra la necesidad de desarrollar nuevos modelos de negocio centrados en servicios y fortalecer los existentes, por cuanto la diversificación en este segmento del sector se ha proyectado con un crecimiento para el año 2030 de cerca de un 30\% [14]. Sin embargo, las perspectivas de futuro y la inclusión de tecnologías de información en los procesos de comunicación y comercialización generarán la necesidad de que los fabricantes gestionen cadenas de valor en las cuales se incluyan clientes, proveedores y socios de cumplimiento de pedidos [21].

\section{DESARROLlo}

\section{A. Transformación de los procesos de operación y administración en la ensambladora y red de concesionarios Honda}

Para el desarrollo del programa de transformación se utilizó el marco de metodología DBT para transformación digital de negocios [8]. La metodología describe 8 etapas, iniciando con la etapa cero denominada gobierno del proyecto, en la cual además de definición del gobierno, se limitó el alcance organizacional del programa de transformación digital a las unidades de negocio relacionadas con HONDA motocicletas, se realizó toda la planeación de entrevistas con los cargos estratégicos y operativos para conocer las prioridades, necesidades y visión del negocio.

En la etapa número uno denominada alineamiento estratégico, se sintetizaron las conclusiones del estudio realizado en el año 2013 en la red de concesionarios de Honda motocicletas, el cual indicaba dos puntos importantes: El primero era que la Red dedicaba una importante cantidad tiempo a actividades contables, administrativas y financieras, que le restaban foco en el desarrollo de sus actividades principales como son comercialización de los vehículos y el servicio posventa de motocicletas.

La siguiente etapa denominada arquitectura empresarial actual, modeló la AE de la ensambladora y los concesionarios en los componentes de sistemas de información, infraestructura técnica, procesos de negocio, roles asignados a los procesos e indicadores (en caso de contar con ellos). Se hizo un especial énfasis en descubrir oportunidades de mejora y tecnología obsoleta ya fuera en sistemas de información o infraestructura que pudiesen influir en la priorización del mapa de ruta.

Posteriormente, se realizó la etapa de análisis de brechas, donde se categorizaron las necesidades clave en términos de negocio y tecnología y se ponderaron los riesgos asociados; adicionalmente, se tomaron decisiones acerca de los posibles escenarios de solución

Una vez realizadas estas etapas se obtuvo información suficiente para diseñar una arquitectura de solución o futura, la cual incluyo la identificación de los procesos y tecnología que podían hacer parte del modelo único replicable (MUR), y una sub-etapa llamada Discovery DBT en la cual se identificaron y se valoraron escenarios de innovación y se adicionaron al mapa de ruta de acuerdo a sus beneficios.

Finalmente, se construyeron escenarios del mapa de ruta, con casos de negocio para respaldar una decisión de implementación la cual fue avalada por la gerencia estratégica de la organización.

\section{RESUltados}

\section{A. Centro de Transformación de Tecnología (CTT)}

Dentro de este proceso, FANALCA creo un grupo interdisciplinario denominado CTT, cuya misión fue definida así: "Habilitar el despliegue de la estrategia mediante la transformación de la arquitectura empresarial a través de iniciativas de innovación que combinan las disciplinas de valor, personas, procesos y tecnología". El CTT de FANALCA se compone de 6 disciplinas como se observa en la Fig. 2: Gestión de personas encargada de elaborar y/o ejecutar los planes de gestión del cambio, comunicación, formación, gestión de interesados y adopción del cambio. Gestión de proyectos (PMO), la cual inicio co-liderando el programa. Business Partner que corresponden a roles técnicos que entienden los procesos de negocio y están constantemente ayudando a concretar sus iniciativas. Arquitectura técnica la cual vela constantemente por los aspectos de tecnología y sistemas. La gestión de valor roles en los cuales se monitorea el desarrollo de la inversión y especialmente la obtención de beneficios y la gestión de procesos realizando todo el modelado de procesos de la transformación y los evolutivos posteriores.

Este grupo inició su labor en el programa, coordinando la interacción y servicio con las áreas de negocio y trabajó de la mano con las firmas consultoras en cada disciplina planteada; posteriormente, tuvo un rol activo en la implementación de las soluciones y actualmente propone y coordina los evolutivos posteriores.

\section{B. Centro de Servicios Compartidos (CSS)}

Se realizó un diseño e implementación de un nuevo centro de servicios compartidos, al cual se llevaron los procesos financieros, contables y de nómina de la red de concesionarios, una vez rediseñados y estandarizados. El diseño incluyo todo el modelo de servicios de CSS, los acuerdos de niveles de servicio y los indicadores de desempeño, posteriormente se implementaron las herramientas tecnológicas que soportaron los procesos diseñados. 


\section{Implementación de una Solución de Gestión del Talento} Humano (HMC)

Siendo los colaboradores de FANALCA uno de sus principales activos y su bienestar una prioridad para la Organización, el proyecto incluyó la revisión de procesos e implantación de una suite de gestión del Talento Humano que incorporó los procesos de selección, reclutamiento, ingreso, contratación, capacitación, aprendizaje, objetivos, desempeño, compensación, sucesión, desarrollo de carrera y nómina. De esta forma se ha buscado implementar la cultura de autogestión entre colaboradores de FANALCA, una reducción significativa en las tareas operativas del área de Talento Humano, obtener información integral de los colaboradores, alineamiento de los objetivos, organización y colaboradores e implementar una compensación variable basada en desempeño individual.

\section{Implementación de una solución de planificación de recursos empresariales (ERP)}

El rediseño de procesos y la centralización de actividades administrativas y contables de la red de concesionarios en el CSS, se habilitó con la implementación de una solución ERP de soporte a la operación, como resultado la red de concesionarios se enfocó en los procesos relacionados con el cliente y se obtuvo una única fuente de datos, ver Fig. 2.

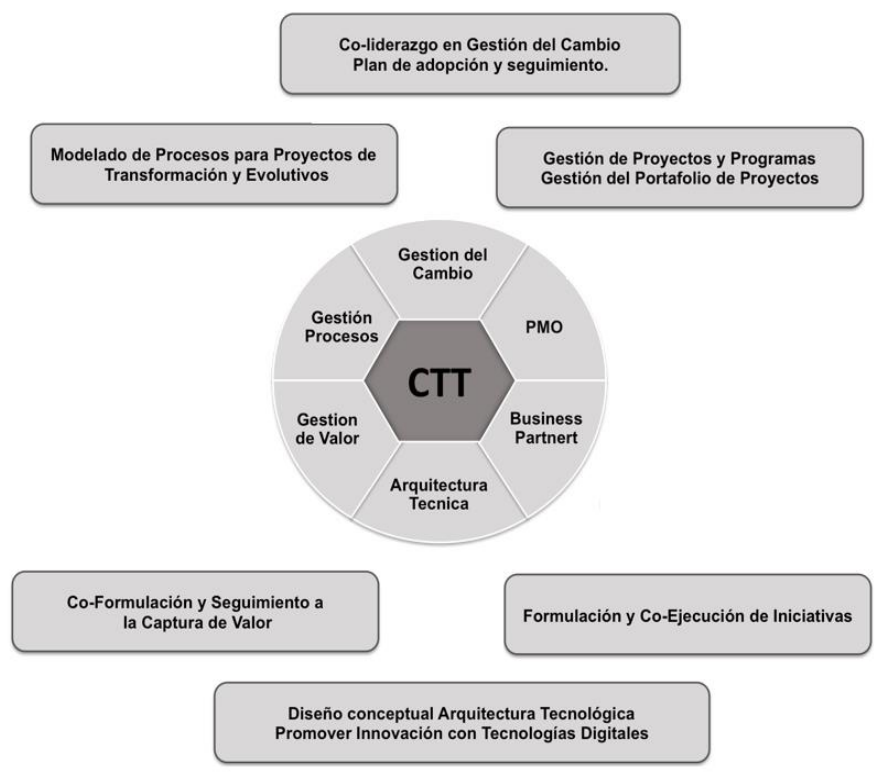

Fig. 2. Esquema de funciones del centro de transformación de tecnología disciplinas y servicios.

\section{E. Implementación de una solución de gestión de} relacionamiento con los clientes (CRM)

El despliegue en la red de concesionarios incluyó también una revisión y mejora de los procesos de mercadeo, ventas y posventa de la unidad de negocio y la implementación de una herramienta CRM Cloud integrada con el BackOffice (ERP) y la matriz FANALCA, permitiendo incrementar la efectividad en diferentes procesos, de acuerdo con lo indicado en la Tabla I.
TABLA I.

IMPACTOS OBTENIDOS A PARTIR DE LA IMPLEMENTACIÓN DEL PROYECTO Y LOS SISTEMAS DE INFORMACIÓN.

\begin{tabular}{|l|l|c|}
\hline \multirow{1}{*}{ Impacto } & \multicolumn{1}{c|}{ Indicadores } & Incremento \\
\hline \multirow{2}{*}{ Prospectos } & Prospectos digitales (\%) & $62 \%$ \\
\cline { 2 - 3 } & Prospectos en almacenes (\%) & $34 \%$ \\
\hline Ventas & Efectividad de cierre de ventas (\%) & $37.8 \%$, \\
\hline
\end{tabular}

F. Diseño de la arquitectura empresarial para las siguientes fases.

Finalmente buscando dar respuestas a las necesidades de otras áreas funcionales y unidades de negocio de la organización, se planteó un roadmap priorizando tres fases de evolución, orientadas a un conjunto de objetivos estratégicos de crecimiento con rentabilidad sustentable. La primera fase de evolución se orienta a la experiencia de cliente final y empresarial, la segunda se busca lograr mayor eficiencia y productividad de la cadena de suministro y la última fase, está enfocada a asegurar la sustentabilidad y el soporte con las funciones de las áreas de servicio.

\section{CONCLUSIONES}

El proyecto permitió la transformación tecnológica en procesos del negocio en la unidad HONDA Motocicletas de FANALCA, aumentado el nivel de satisfacción de servicio al cliente gracias a procesos organizacionales eficientes y rápidos que permiten brindar un servicio integral a los usuarios de la marca HONDA.

$\mathrm{Al}$ inicio de este proyecto se identificó que la calidad de datos en la Red de Concesionarios era del $44 \%$ en la información relacionada con clientes y un $73 \%$ en la información del producto. Por tanto, se diseñaron integraciones tomando como origen la ensambladora en la creación de productos y como fuente la Red de concesionarios. Una vez realizadas las integraciones expuestas a través de un bus de datos o a través de Web-Services, se definió un procedimiento de manejo de errores y de excepciones creado entre la Ensambladora y la Red de Concesionarios que define tanto el proceso en los sistemas de información, como el proceso de negocio. La construcción de todas las integraciones, creo un desafío para el grupo de desarrollo de FANALCA, debido a los cambios que debían hacerse a los sistemas legados para optimizar y mantener la integridad de los datos enviados.

El proceso de transformación generó un esfuerzo continuo de capacitación al nuevo personal y refuerzo en las mejores prácticas tanto en FANALCA como en la red de distribución, generando por esta vía, la transformación de la Red de concesionarios que incluyó una revisión y mejora de los procesos de mercadeo, ventas y posventa.

El personal de FANALCA se vio beneficiado al poder acceder a capacitaciones, logrando una alta cultura de autogestión apoyada en los sistemas de información, reduciendo tareas operativas y aumentando la confiabilidad de la información.

\section{REFERENCIAS}

[1] ANDI, «Las motocicletas en Colombia: aliadas del desarrollo del país,» Bogotá, 2017.

[2] OECD, «Oslo Manual 2018: GUIDELINES FOR COLLECTING, REPORTING AND USING DATA ON INNOVATION 4TH EDITION. The Measurement of Scientific, Technological and Innovation Activities,» Paris/Eurostat, Luxembourg., 2018. 
[3] GARTNER, «Gartner IT Glossary > Digital Business Transformation,» 2018. [En línea]. Available: https://www.gartner.com/it-glossary/digitalbusiness-transformation.

[4] O. Fuente, «¿Qué es la Transformación Digital y cómo crear Digital Business?,» Enero 2018. [En línea]. Available: https://www.iebschool.com/blog/que-es-transformacion-digital-business/. [Último acceso: 2018].

[5] D. Marchand y M. Wade, «Digital Business Transformation: Where is your company on the journey?,» IMD PERSPECTIVES FOR MANAGERS, pp. 1-4, 2014.

[6] M. McRobbie, «Innovation, Transformation, Community,» Internet2, Arlington, VA, 2012.

[7] IDG, «"State of Digital Business Transformation" White Paper,» 2018. [En línea]. Available: https://www.idg.com/tools-for-marketers/2018state-of-digital-business-transformation-white-paper/.

[8] R. Fish, «Digital Business Transformation: Qué es y qué significa para las empresas,» 2016. [En línea]. Available: http://gatalks.grupoassa.com/es/01-DBT/\#!digital-businesstransformation.

[9] MIT CISR, «Research: Research-overview, Classic-topics, Enterprisearchitecture,» 2017. [En línea]. Available: https://cisr.mit.edu/research/research-overview/classic-topics/enterprisearchitecture/.

[10] M. Rothman, E. Murphy, «Data mining: a practical approach for database marketing,» IBM White Paper, Dallas, 1995.

[11] S.-H. Li, D. C. Yen, W-H Lu., C. Wang, «Identifying the signs of fraudulent accounts using data mining techniques,» Computers in Human Behavior, pp. 1002-1013, 2012.

[12] SAP AG, «SAP Predictive Analysis,» SAP Group, Walldorf, 2014.

[13] J. Han, M. Kamber y J. Pei, Data Mining, Concepts and Techniques, Waltham: Morgan Kaufmann, 2012.

[14] ANDI-INNPULSA-VTSAS, «CIERRE DE BRECHAS DE INNOVACIÓN \& TECNOLOGÍA,» ANDI-INNPULSA-VTSAS, Medellin (Colombia), 2018.

[15] M. Muffatto y A. Parayo, «Implementation of e-procurement and efulfillment processes: A comparison of cases in the motorcycle industry,» International Journal of Production Economics, p. 339-351, 2004.

[16] Motorcycle Industry Association, «The UK Motorcycle Industry. Manufacturing, Public Policy, The Economy and Growth,» The Motor Cycle Industry Association Limited, Allesley (UK), 2012.

[17] GHK, «The Economic Significance of the UK Motor Cycle Industry,» The Motor Cycle Industry Association, London, 2010.

[18] G. Reed, V. Story y V. Saker, «Information technology: changing the face of automotive retailing?,» International Journal of Retail \& Distribution Management, vol. 32, $\mathrm{n}^{\circ}$ 1, p. 19 - 32, 2004.

[19] S. Rao , «A Review on Competitive Structure of Automobile Industry,» Asian Journal of Applied Science and Technology, vol. 1, $\mathrm{n}^{\circ}$ 9, pp. 175$185,2017$.

[20] EYGM Limited, «Automotive retail 2030 - Evolution of dealerships and potential new roles in retail,» EY, 2018.

[21] T. JELASSI y S. LEENEN, «An E-Commerce Sales Model for Manufacturing Companies: A Conceptual Framework and a European Example,» European Management Journal, vol. 21, $\mathrm{n}^{\circ}$ 1, pp. 38-47, 2003.

[22] P. Wells, «Sustainable business models and the automotive industry: A commentary,» IIMB Management Review, vol. 25, $\mathrm{n}^{\circ}$ 4, pp. 228-239, 2013.

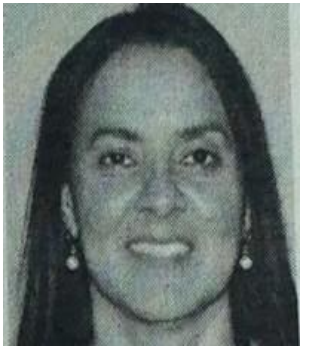

Mónica Vélez O. Ingeniera de sistemas de la Universidad de San Buenaventura, con más de 14 de años de experiencia en el desarrollo de sistemas de información y arquitectura empresarial en el sector automotor. Recibió certificaciones internacionales en ITIL 2011 Foundation y Oracle Database 9i Certification Associate. Su experiencia profesional se ha centrado en el desarrollo de sistemas de información, su mantenimiento, gerenciamiento y el desarrollo de nuevos proyectos especializados para una gran empresa del sector automotor en Colombia. Coinvestigadora en el proyecto, responsable de la interacción entre áreas, generando gestión eficiente de la arquitectura organizacional.

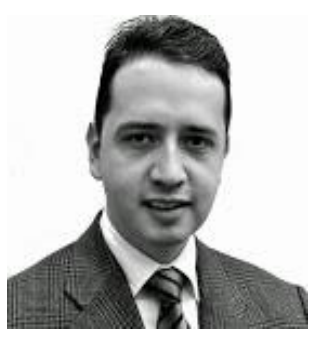

Hermann Stuart Fuquen Gonzalez. Es Ingeniero Industrial de la Universidad de la Sabana en Colombia, graduado en la Maestría en Administración y Economía de la Innovación en la Universidad Tecnológica de Chalmers en Göteborg, Suecia. Durante 4 años estuvo vinculado a COLCIENCIAS como asesor del Programa de Desarrollo Tecnológico Industrial y Calidad, donde realizó seguimientos a un alto volumen de proyectos empresariales que aplicaban a los distintos instrumentos de apoyo a la innovación que el gobierno colombiano. Posee experiencia académica en la Universidad de la Sabana, Universidad Sergio Arboleda y fue investigador de la Fundación Universitaria los Libertadores, posee formación en temas de Propiedad Intelectual a través diversos cursos nacionales e internacionales dictados por WIPO. Actualmente es consultor de innovación Tecnológica para proyectos de innovación desarrollados por empresas de distintos sectores. ORCID: 0000-0001-7692-4216

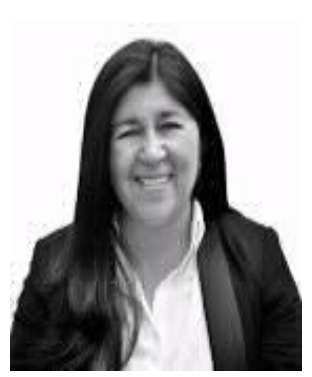

Claudia Marcela Sánchez. Ingeniera mecánica de la Universidad Nacional de Colombia, Especialista en Innovación y Desarrollo de Negocios y Master en Gerencia de la Innovación Empresarial de la Universidad Externado de Colombia. Su experiencia profesional inició siendo asesora técnica del Programas Nacionales de Desarrollo Tecnológico Industrial y Calidad y posteriormente del Programa Nacional de Investigaciones en Energía y Minería en el área de Innovación en COLCIENCIAS, realizó seguimiento a proyectos de investigación, desarrollo tecnológico e innovación. Posee experiencia docente en la Universidad Nacional de Colombia y en la Universidad Externado de Colombia, donde actualmente es tutora de trabajos de grado del MBI. Actualmente es consultora de en innovación tecnológica para grandes empresas de distintos sectores.

ORCID: 0000-0002-1090-7541 